\title{
Determinación de la curva de retención de agua para relaves multimetálicos de la industria minera de Chile
}

Determination of the soil-water characteristic curve for multimetal tailings of the mining industry of Chile

Fecha de entrega: 6 de diciembre 2018 Fecha de aceptación: 29 de abril 2019

\section{Jaime Musso ${ }^{1}$ y Gonzalo Suazo ${ }^{2}$}

${ }^{1}$ Laboratorio de Ensaye de Materiales y Control de Obra LEMCO, Universidad Técnica Federico Santa María, Lab 313, Av. España \#1680, Valparaíso, Chile, jaime.musso.hott@gmail.com

${ }^{2}$ Departamento de Ingeniería Civil, Universidad Técnica Federico Santa María, Avenida España 1680, Valparaíso, Chile, gonzalo.suazo@usm.cl

Los depósitos de relaves están expuestos a una serie de fenómenos que promueven su desaturación y su evolución a una condición parcialmente desaturada, e.g. procesos de infiltración y generación de frentes de evaporación. Lo anterior se traduce en la generación de presiones de poros negativas en los espacios intersticiales lo que condiciona la tasa de flujo de agua y oxígeno, las tasas de evaporación y la estabilidad física de la estructura (resistencia al corte). Para poder caracterizar estos fenómenos es necesario conocer la curva de retención de agua (SWRC), que relaciona gráficamente el estado de humedad y la succión desarrollada en el material. La obtención de esta curva, especialmente para suelos finos, requiere extensos periodos de tiempo para su determinación, por lo que en la práctica se tienden a estimar estas curvas a partir de modelos de estimación aproximados no disponibles para relaves. Considerando lo anterior, el presente estudio contempla la caracterización y obtención de la SWRC de 13 muestras de relaves provenientes de la industria minera nacional y se propone un modelo de estimación simplificado de la SWRC a partir del contenido de finos, humedad de saturación y la gravedad específica de los relaves. Este modelo facilita la caracterización de fenómenos de respuesta no saturados en etapas de ingeniería básica. De manera adicional, se presenta la permeabilidad parcialmente saturada de estos materiales.

Palabras clave: curva de retención de agua, succión, relaves, suelo no saturado
Tailings storage facilities are exposed to a series of phenomena that promote the desaturation of the materials, e.g. seepage and evaporation fronts. The desaturation generates negative pore pressure, the so-called suction, in the pore space, which controls unsaturated seepage, transport of solutes, and shear strength of the facility. If these phenomena need to be characterized, the Soil Water Retention Curve (SWRC) is needed. This curve relates water content and suction. The construction of the SWRC in fine-grained soils requires significant equalization times, when using conventional direct methods, which limits its routine determination. Therefore, practitioners use approximate models, which are not commonly available for tailings. Regarding this, the present study explores SWRC determination in tailings samples recover from 13 tailings in Chile. A simplified estimation model is proposed based on basic properties of the material, e.g. fines content, saturation water content and the soil's specific gravity. This type of model will allow for the construction of the SWRC in the early development of engineering for tailings. In addition to this, unsaturated permeability of the tailings is characterized.

Keywords: soil water retention curve, suction, tailings, unsaturated soil

\section{Introducción}

A diferencia de los suelos saturados y los suelos secos, los suelos parcialmente saturados están constituidos por tres fases: la fase sólida, líquida y gaseosa. La interacción del agua y aire en los espacios vacíos generan tensiones intersticiales negativas o succión (Fredlund y Rahardjo, 
1993). El estudio de suelos parcialmente saturados presenta una alta complejidad y un nivel de desarrollo científico e ingenieril limitado en comparación al nivel de conocimientos en la respuesta de suelos en estados límites, i.e. seco o saturado (Fredlund, 2000). Con el desarrollo de las tecnologías, las barreras de cálculo computacional que antiguamente limitaron el desarrollo de la ingeniería de suelos parcialmente saturados ha prácticamente desaparecido, y por tanto, se ha avanzado en la comprensión de fenómenos como flujos no saturados e infiltración, respuesta al corte bajo succión, estabilidad de suelos naturales y en el diseño de elementos de control de contaminantes, e.g. barreras capilares (Fredlund, 2000). El estudio de las propiedades de suelos parcialmente saturados se suele basar en la definición de la curva de retención de agua (SWRC por su sigla en inglés) para cada material, i.e. curva que establece la relación entre la succión matricial y el contenido de humedad o grado de saturación de un suelo (Mahmood y Kareem, 2010). Esta curva permite determinar otras propiedades básicas de los suelos como lo son la permeabilidad no saturada y caracterizar fenómenos avanzados como la resistencia de corte y cambios volumétricos.

Los depósitos de relaves están sometidos a una serie de fenómenos no saturados como lo son la infiltración de agua hacia el subsuelo y la superficie, la generación de frentes de evaporación, el agrietamiento de relaves integrales o los efectos de la succión en procesos de erosión. Si bien estos fenómenos han recibido una baja atención en el pasado, se espera que se incorporen en etapas de diseño y verificación de estabilidad física-química de los depósitos. Por tanto, existe la necesidad de avanzar hacia la caracterización de la SWRC para relaves provenientes de la industria minera nacional que permitan a ingenieros contar con información preliminar para el desarrollo de ingeniería básica. En este contexto, el presente estudio caracterizara la SWRC de 13 relaves provenientes de la industria del hierro, cobre, molibdeno y oro. Esta recopilación incluye arenas de relaves, lamas y relaves integrales. Por último, se propone un método simplificado para la obtención de la SWRC a partir de parámetros básicos de los relaves.

\section{Curva de retención de agua}

La curva de retención de agua SWRC, como representación gráfica de la relación entre la succión del suelo y su contenido de humedad, representa el estado energético del suelo para una determinada humedad o grado de saturación (Mahmood y Kareem, 2010). Esta curva, constituye la capacidad de conservación de agua de un suelo ante distintos estados de succión, presentando tres zonas claramente identificables y divididas por dos puntos, como se puede observar en la Figura 1a, conocidos con el nombre de punto de entrada de aire o air entry value (AEV), y el punto de succión residual (Aubertin et al., 1998). Además, la SWRC tiene comportamientos particulares según la granulometría de la muestra analizada como se presenta en la Figura 1b, donde los suelos finos presentan mayores succiones que los suelos gruesos a una misma humedad.

a)
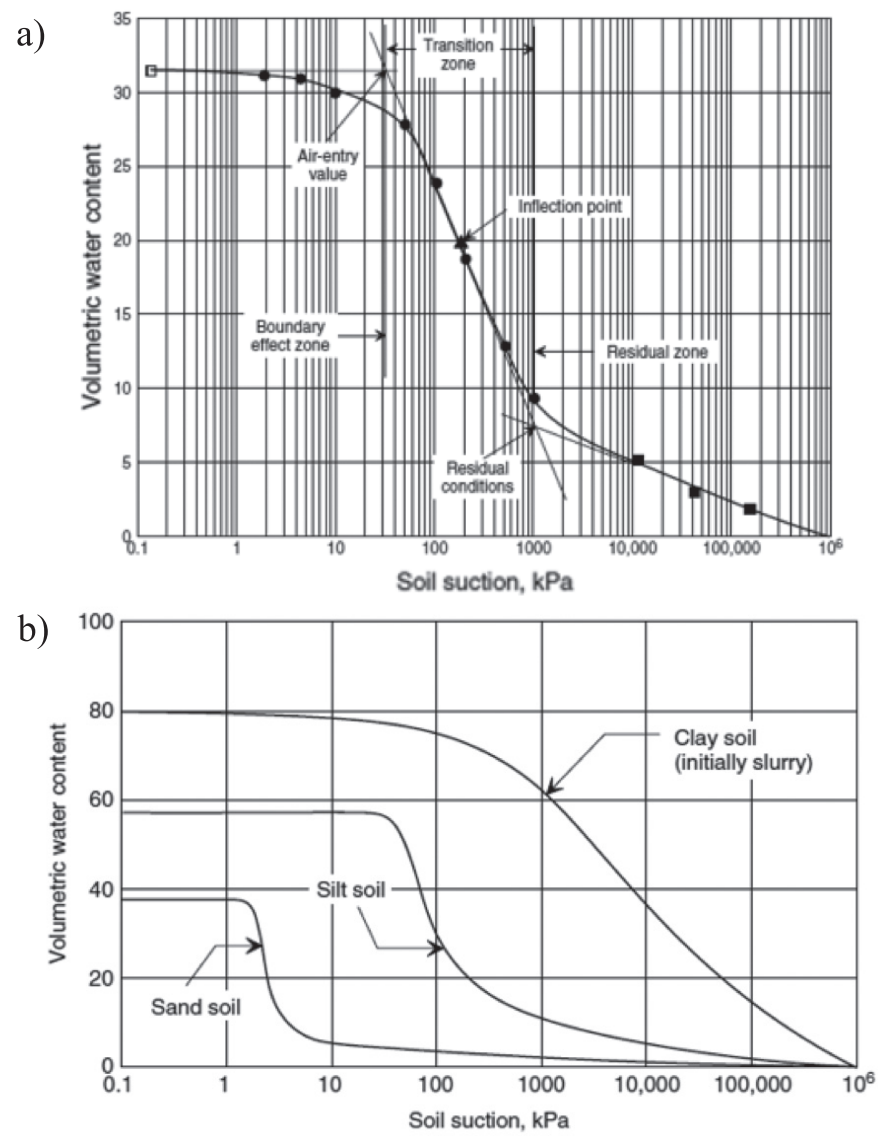

Figura 1: a) Puntos y zonas características de la SWRC y b) forma típica de la SWRC para arenas, limos y arcillas (Fredlund y Rahardjo, 1993)

\section{Caracterización de relaves}

Se estudiaron las SWRCs de 13 relaves de la industria minera nacional mediante cámara de presión y platos cerámicos porosos estandarizado bajo la norma ASTM D6836 (2002). Además, se realizó una completa 
caracterización geotécnica de las muestras de relaves. Los relaves analizados mostrados en la Figura 2, provienen de tranques y embalses de relaves como así también de depósitos de relaves en pasta y filtrados de la industria del cobre, molibdeno, oro y hierro. La información particular de estos depósitos de relaves se presenta en la Tabla 1.
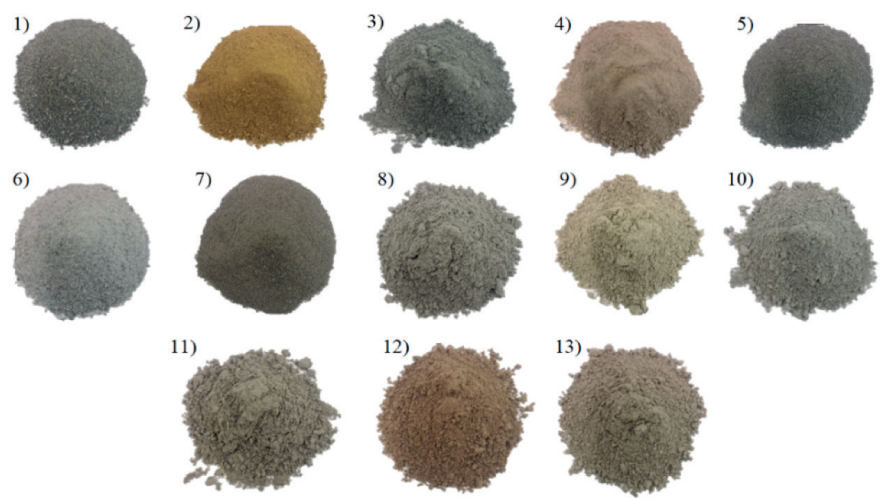

Figura 2: Relaves utilizados en el presente estudio geotécnico.

Para la caracterización de los relaves, se realizaron ensayos de gravedad específica, densidad mínima, Proctor estándar, granulometría por tamizado y sedimentación, límites de Atterberg y permeabilidad saturada, siguiendo normativa
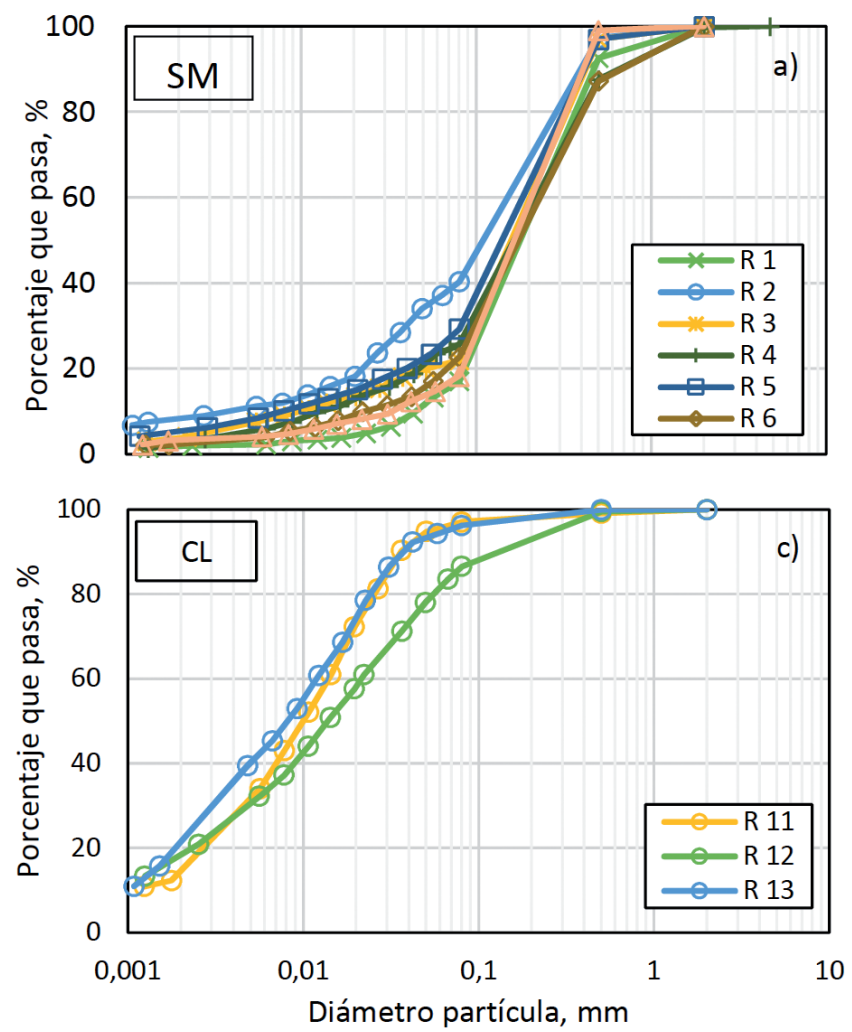

chilena y ASTM. Los resultados de esta caracterización geotécnica se presentan de forma resumida en la Tabla 1. A partir de clasificación USCS, se identifican tres grupos o tipos de relaves (SM, ML-CL, CL) y cuyas distribuciones granulométricas se presentan en la Figura 3.

\section{Análisis de la SWRC obtenidas mediante la técnica de traslación de ejes}

Las curvas de retención de agua fueron obtenidas mediante una cámara de presión bajo la técnica de traslación de ejes (Riquelme y Godoy, 2016). La cámara y el sistema de control de presión fueron desarrollados e implementados en los laboratorios de Geotecnia de la UTFSM (LEMCO). Los valores experimentales de la SWRC fueron obtenidos mediante la medición de cinco puntos característicos de forma equidistante en una gráfica logarítmica, i.e. 0.1, 30, 100,400 y $1500 \mathrm{kPa}$, donde el segundo y último punto corresponden aproximadamente a los valores del punto de la entrada de aire AEV y succión residual de la SWRC. Los tiempos de equilibrio (equalization time) fueron en general superiores a 7 días. El primer punto de contenido de agua de la curva ( $0.1 \mathrm{kPa}$ para reflejar succión nula) corresponde al contenido gravimétrico de humedad al límite de saturación
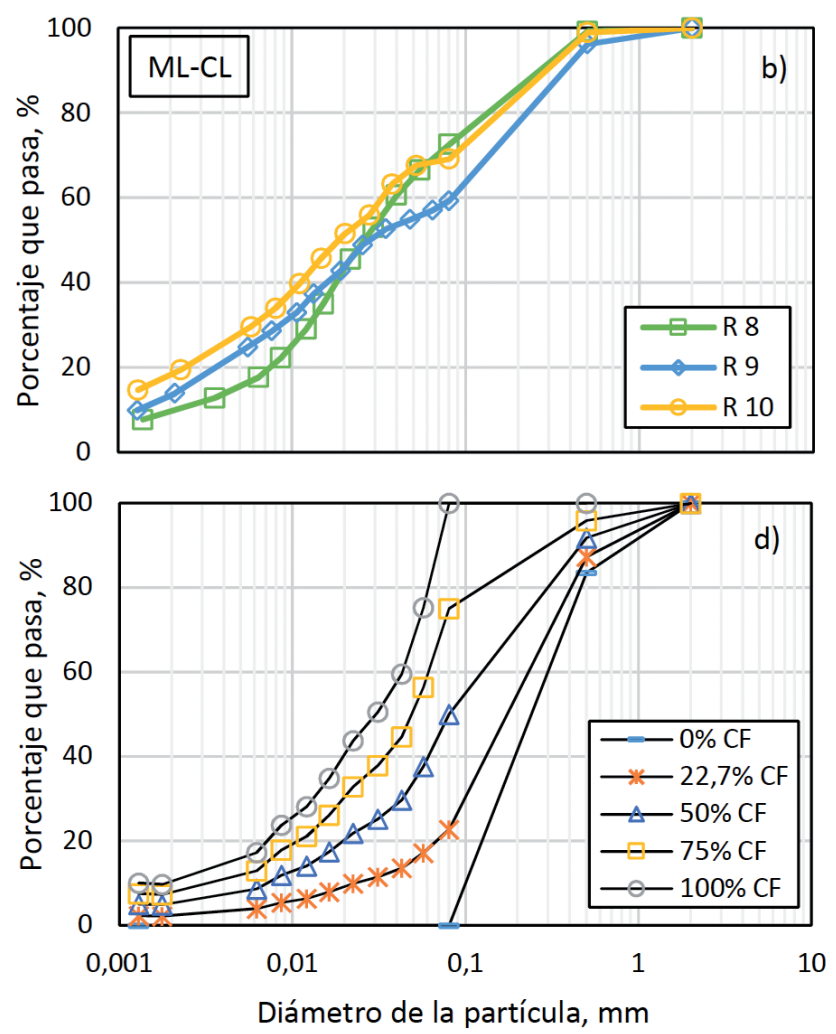

Figura 3: Granulometrías para relaves: a) con clasificación SM, b) con clasificación ML-CL, c) con clasificación CL y d) relaves sintéticos a partir de relave $\mathrm{N}^{\circ} 6$ para calibración de los modelos de estimación. 
Tabla 1: Resumen caracterización relaves multimetálicos provenientes de la industria chilena.

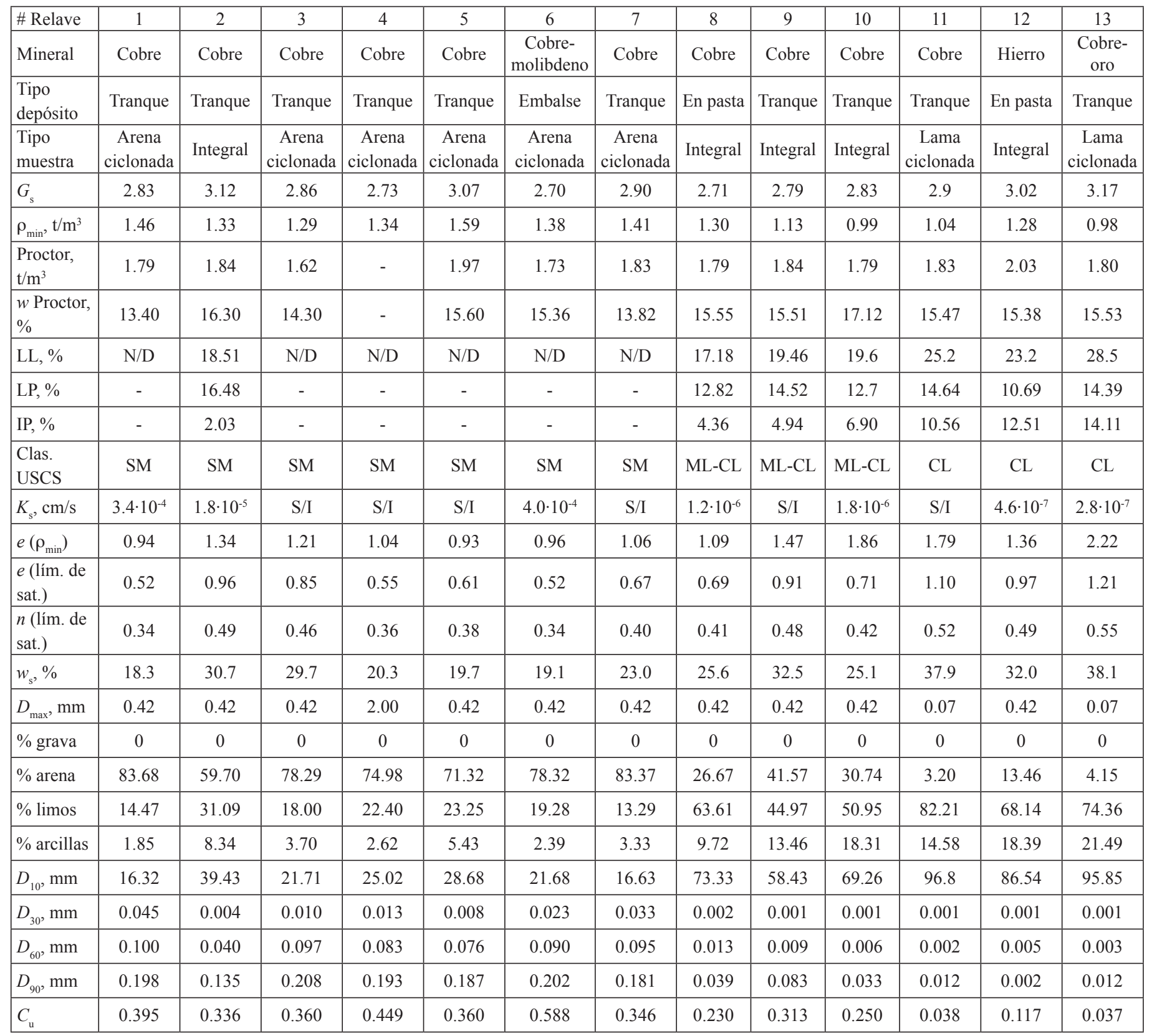

$\left(w_{\mathrm{s}}\right)$, determinado de manera experimental y que se resume en la Tabla 1.

Para la representación de los puntos en una curva única y continua, los valores experimentales fueron inicialmente ajustados mediante diversos modelos de regresión, como lo son van Genuchten (1980), Fredlund y Xing (1994) y Pham y Fredlund (2011). Sin embargo, el modelo que mejor representa el comportamiento de la SWRC en relaves resultó ser Fredlund y Xing (1994), lo que es consistente con otras investigaciones en relaves (Suazo et al., 2016), y que relaciona la humedad gravimétrica del material $(w)$ con el contenido de agua de saturación $\left(w_{\mathrm{s}}\right)$, la succión $(\psi)$, y succión residual $\left(\psi_{\mathrm{r}}\right)$ del suelo, según (1). Los parámetros $a, m y n$ son parámetros de ajuste que eventualmente podrían tener un significado físico (Suazo et al., 2016).

$$
w(\psi, a, n, m)=\frac{w_{s}}{\left(\ln \left[e+\left(\frac{\psi}{a}\right)^{n}\right]\right)^{m}} *\left[1-\frac{\ln \left(1+\frac{\psi}{\psi_{r}}\right)}{\ln \left(1+\frac{1.000 .000}{\psi_{r}}\right)}\right]
$$

De la Figura 4 se puede observar que los relaves constituidos principalmente por arenas poseen un quiebre asociado al $\mathrm{AEV}$ a una succión menor que para los relaves más finos, 
lo que es esperable debido a que las mayores presiones intersticiales se generan en materiales más finos. Se debe mencionar que el AEV representa el punto para el cual comienza a ingresar aire a la matriz de suelo en el proceso de desaturación. La ubicación de este punto se presenta en la Figura 5a.

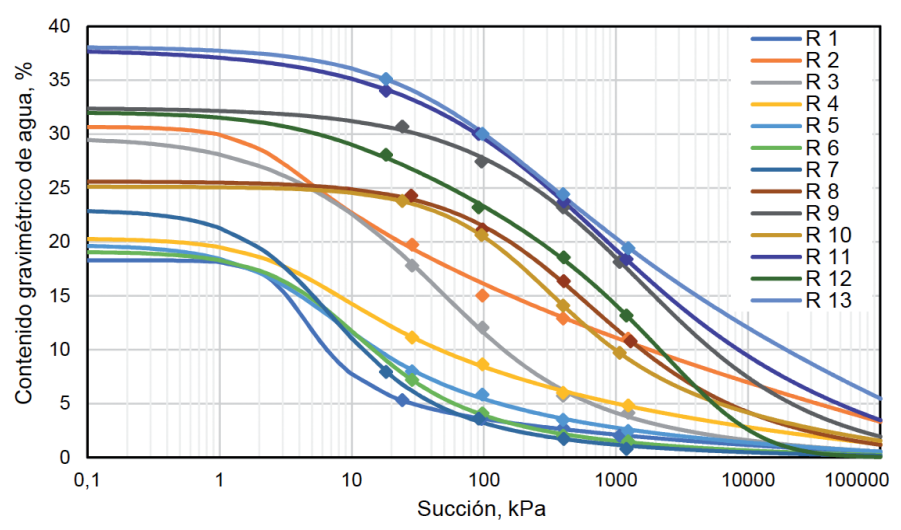

Figura 4: Dispersión de valores experimentales y ajuste mediante modelo de Fredlund y Xing (1994) para relaves provenientes de la industria minera de Chile

a)

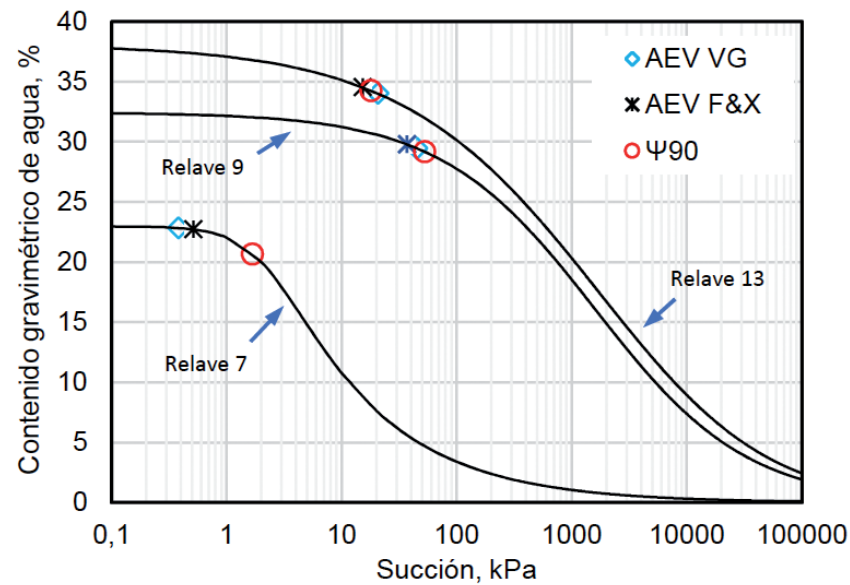

b)

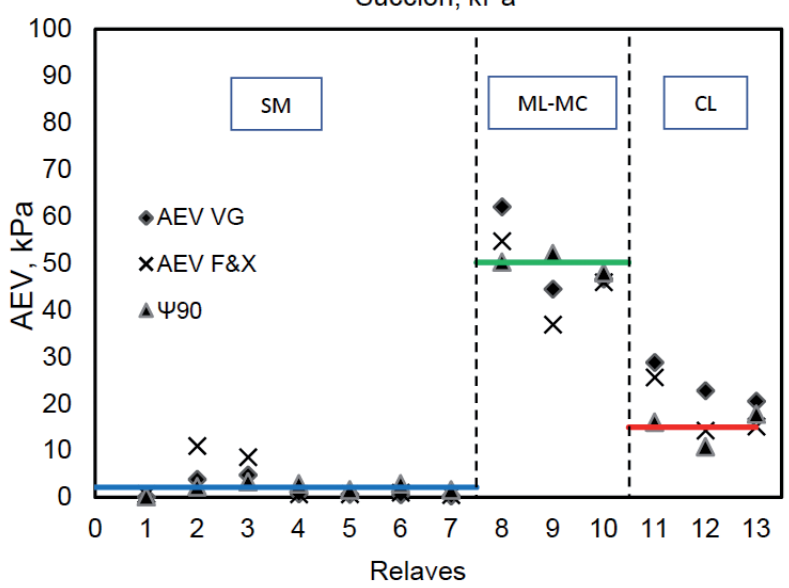

Figura 5: a) Ubicación de los AEV en tres relaves de tipo SM, ML-CL y CL de la industria nacional y b) distribución de las magnitudes de los AEV para los 13 relaves en estudios según clasificación
Algo similar ocurre con la pendiente de la curva en la zona de desaturación, la que es significativamente menor en relaves más finos (lamas), como resultado de una mayor demanda de tensión a un contenido de humedad dado. La determinación del punto de la entrada de aire AEV depende del método de ajuste seleccionado, e.g. van Genuchten et al. (1991) o Fredlund y Xing (1994). En la Figura 5b se presenta el valor de AEV para ambos modelos $\left(\psi_{V G}, \psi_{F X}\right)$ considerando la clasificación general de los tipos de relaves estudiados. Además, se incluye el valor de $\psi_{90}$ (succión al $90 \%$ del contenido de agua de saturación) que en general representa una buena y simple aproximación al AEV.

\section{Conductividad hidráulica parcialmente saturada}

El estudio de la permeabilidad no saturada es la base para el estudio de flujos no saturados en depósitos de relaves, e.g. flujo de drenaje ácido una vez que los depósitos se exponen al aire y agua. Una buena aproximación de esta curva se obtiene a partir de la SWRC y el coeficiente de conductividad hidráulica saturado $\left(K_{s}\right)$ del material utilizando la expresión (2) propuesta por van Genuchten (1980) y validada por Khire et al. (2000):

$$
\frac{K_{\psi}}{K_{s}}=\frac{\left\{1-(\alpha \psi)^{n-1}\left[1+(\alpha \psi)^{n}\right]^{-m}\right\}^{2}}{\left[1+(\alpha \psi)^{n}\right]^{m / 2}}
$$

donde $\alpha, m$ y $n$ son los parámetros de regresión del modelo de van Genuchten y $K_{\psi}$ es la conductividad hidráulica no saturada. La ecuación (2) ha sido ajustada a las SWRCs de los relaves 1 y 10 en la Figura 6. Se puede observar que la conductividad hidráulica decae rápidamente luego de que se excede la succión al AEV. Lo anterior ocurre a succiones bajo $10 \mathrm{kPa}$ para arenas y $100 \mathrm{kPa}$ para limos. En todo el rango de succiones, $K_{\psi}$ es menor para limos que para arenas, lo que se traduce en una mayor dificultad del líquido para fluir en relaves tipo lamas o integrales.

\section{Propuesta de modelo de estimación rápida de SWRC en relaves}

Se propone un modelo de estimación rápida de la curva de retención de agua para estudios de ingeniería básica a partir de la humedad de saturación $\left(w_{s}\right)$ y el contenido de finos CF del material. Se propone además una segunda 
a)

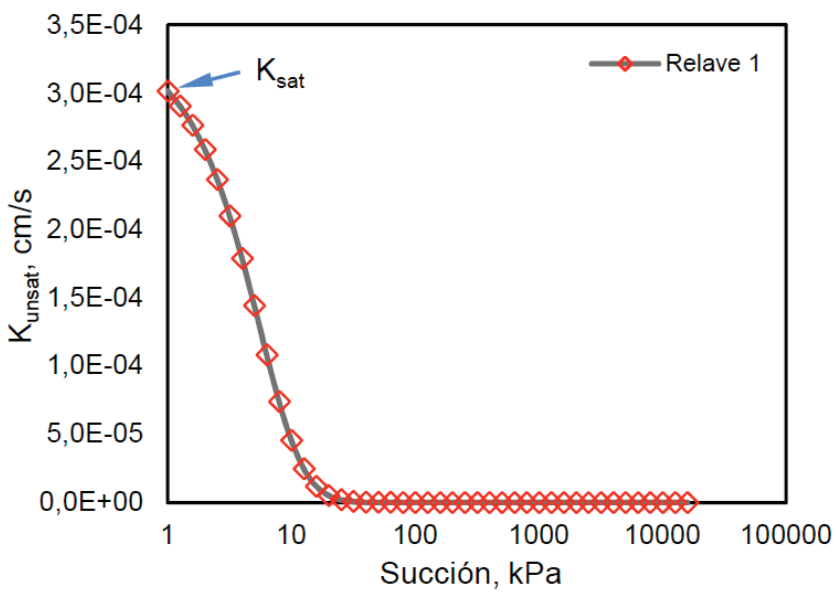

b)

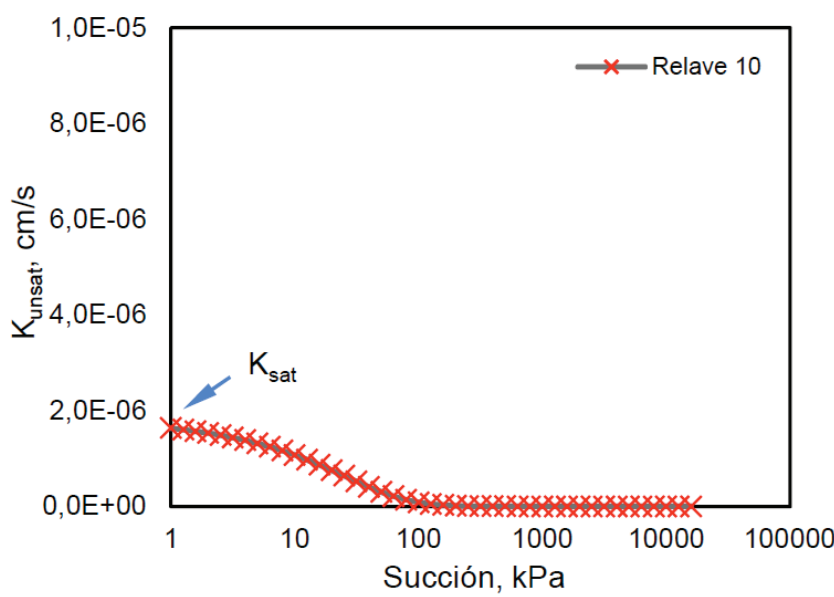

Figura 6: Conductividad hidráulica parcialmente saturada para: a) relave $1 \mathrm{y}$ b) relave 10

simplificación para la obtención de $\left(w_{s}\right)$ a partir de la gravedad específica de los sólidos $G_{s}$ y CF. El modelo de estimación que se desarrolla en este apartado, se construye a partir de la regresión de los parámetros del modelo de van Genuchten $\left(\alpha_{V G}, m_{V G}, n_{V G}\right)$.

Asumiendo una succión residual nula, i.e. modelo 5 de Vereeken et al. (1989), presentada en la ecuación (3), y bajo la hipótesis que la SWRC en relaves está fuertemente condicionada por la cantidad de finos presente en el material, se construyeron granulometrías sintéticas (i.e. se modificó su CF) con muestras del relave 6, lo que resultó en granulometrías con CF de 0, 23, 50, 75 y 100\%, mostradas en la Figura 3d. Para estas cinco muestras se obtuvieron experimentalmente sus SWRCs, según se presenta en la Figura 7. La variación de los parámetros de regresión con el CF se ajustó mediante polinomios de segundo grado lo que arrojó coeficientes de correlación cercanos a la unidad:

$$
\begin{gathered}
w(\psi, a, n, m)=\frac{w_{s}}{\left(1+\left[\frac{\psi}{a}\right]^{n}\right)^{m}} \\
\alpha_{V G}=0,0233 C F^{2}-0,306 C F+0,3767 \\
m_{V G}=0,00007 C F^{2}+0,001 C F+0,1395 \\
n_{V G}=0,0003 C F^{2}-0,0517 C F+3,6221 \\
w_{s}=0,15 C F+12 G_{s}-15,53
\end{gathered}
$$

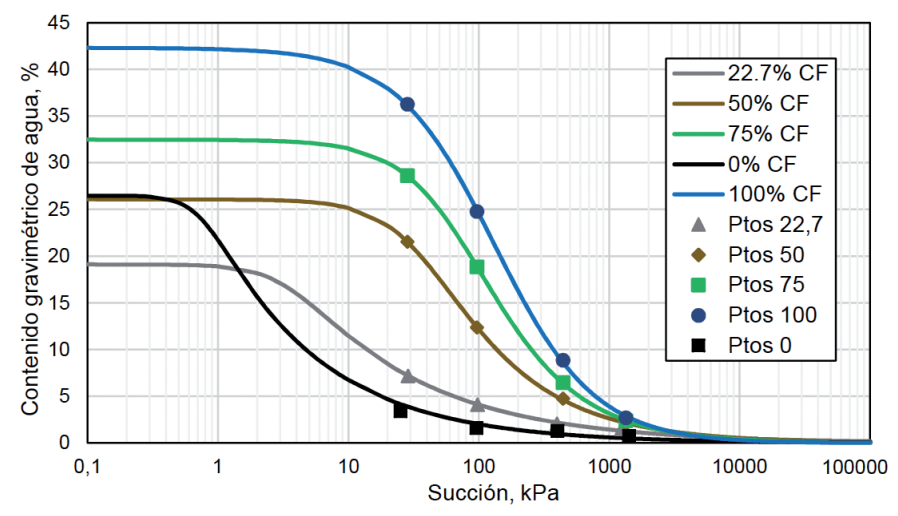

Figura 7: SWRCs provenientes del relave 6 con granulometrías sintéticas.

Con la obtención de los parámetros antes presentados y la humedad de saturación es posible estimar la SWRC para un relave dado. Por otro lado, un análisis de significancia de propiedades de los relaves arrojó que $w_{s}$ se relaciona adecuadamente con $G_{\mathrm{s}}$ y $\mathrm{CF}$, según se presenta en la Figura 8 , y siguiendo una relación lineal de la forma presentada en la ecuación (7).

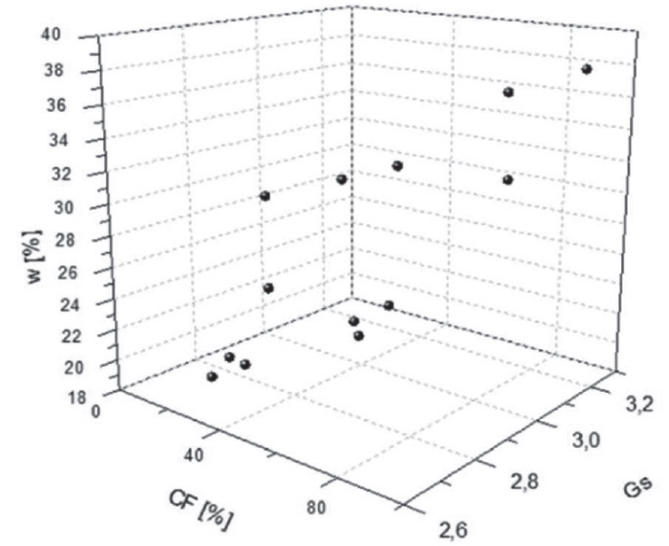

Figura 8: Relación experimental entre el contenido de finos CF, gravedad específica $G_{\mathrm{s}}$ y humedad de saturación $w_{s}$. 


\section{Aplicación de modelo}

Para observar el comportamiento del modelo simplificado de estimación, se seleccionó una SWRC obtenida en relaves por los autores Riquelme y Godoy (2016), de modo de evaluar el rendimiento en un set experimental diferente al utilizado en este estudio. La Figura 9 presenta estos resultados. La Figura 9a muestra el ajuste del modelo cuando se conoce $\left(w_{s}\right)$, mientras que la curva de la Figura $9 \mathrm{~b}$ presenta la curva estimada a partir de parámetros básicos del relave $\left(G_{s}, C F\right)$. Se observa que el grado de ajuste es satisfactorio en ambos casos, pese al uso de propiedades índice básicas de los relaves. Sin embargo, se debe mencionar que estas muestras se prepararon a contenidos de sólidos del 70\% y no consideran los efectos de la presión osmótica. Las modificaciones al modelo para otros contenidos de sólidos están siendo investigados por los autores.

a)

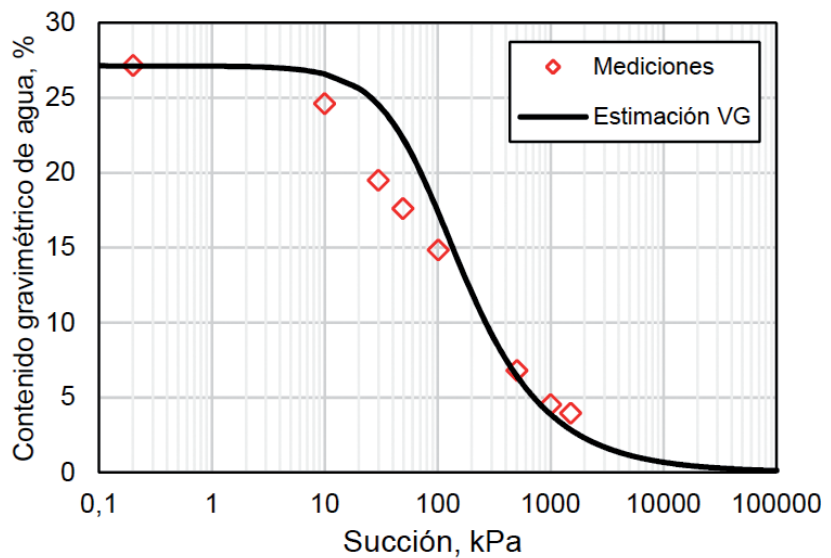

b)

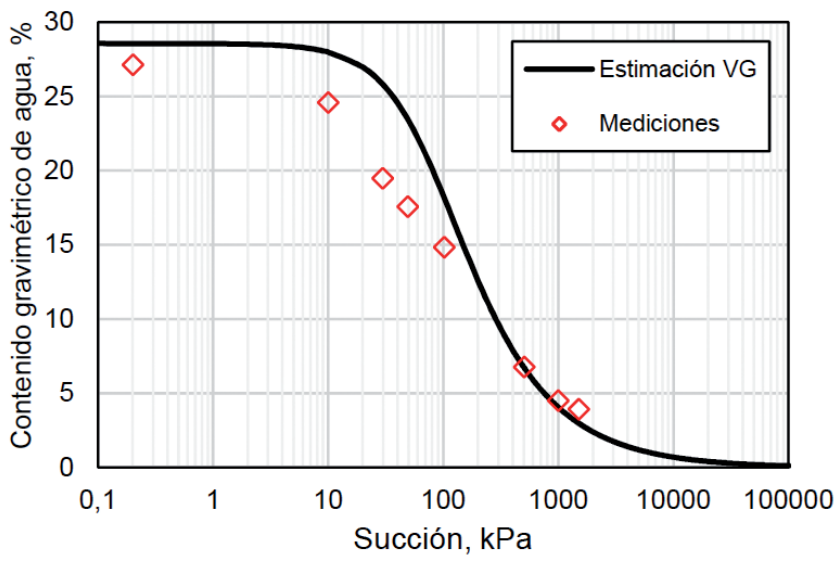

Figura 9: Estimación mediante: a) modelo 1 y b) modelo 2 propuestos, para relave 4 de Riquelme y Godoy (2016)

\section{Conclusiones}

Se realizó la caracterización de 13 relaves provenientes de la industria minera chilena, cuyo origen mineralógico proviene de la extracción de hierro, cobre, molibdeno y oro. Los valores difieren entre sí principalmente por su granulometría. Se determinaron, además, las curvas de retención de agua asociadas a los relaves en estudio compuestas por 7 relaves clasificados como arenas, 3 relaves clasificados como limos y 3 como arcillas, donde las curvas respectivas cambian sus características principalmente por sus características granulométricas de cada relave (e.g. AEV, permeabilidad). Se calcularon las curvas de conductividad hidráulica no saturada para los 13 relaves en estudio, concluyendo que la permeabilidad decae abruptamente luego del punto de entrada de aire respectivo, es decir, a succiones bajo $10 \mathrm{kPa}$ para arenas y $100 \mathrm{kPa}$ para limos.

Finalmente se proponen 2 modelos de estimación de la curva de retención de agua a partir del modelo de van Genuchten, donde se calibran sus parámetros a partir de relaves con granulometrías sintéticas a distintos contenidos de finos. De los modelos propuestos, el modelo 1 contempla la obtención de la SWRC a partir de la humedad de saturación y el contenido de finos CF, mientras que el modelo 2, contempla la obtención de la SWRC a partir del contenido de finos y la gravedad específica. Estos modelos propuestos ahorran gran cantidad de recursos para la obtención de la SWRC, ya que se obtienen a partir de ensayos básicos de laboratorio, por lo cual es posible estimar una curva aceptable en un corto periodo de tiempo, permitiendo entre otras cosas, establecer un modelamiento del flujo al interior de estructuras geotécnicas y, además, tener conocimiento del comienzo de la producción de drenaje ácido.

\section{Referencias}

ASTM D6836 (2002). Determination of the soil water characteristic curve for desorption using a hanging Column, pressure extractor, chilled mirror hygrometer, and/or centrifuge. ASTM International, West Conshohocken, PA, USA

Aubertin, M., Ricard J.F. and Chapuis, R.P. (1998). A predictive model for the water retention curve: application to tailings from hard-rock mines. Canadian Geotechnical Journal 35(1), 55-69 
Fredlund, D.G. (2000). The implementation of unsaturated soil mechanics into geotechnical engineering. Canadian Geotechnical Journal 37(5), 963-986

Fredlund, D.G. and Xing, A. (1994). Equations for the soil-water characteristic curve. Canadian Geotechnical Journal 31(4), $521-532$

Fredlund, D.G. and Rahardjo, H. (1993). Soil mechanics for unsaturated soils. Wiley, New York

Khire, M.V., Benson, C.H. and Bosscher, P.J. (2000). Capillary barriers: design variables and water balance. Journal of Geotechnical and Geoenvironmental Engineering 126(8), 695 $-708$

Mahmood, K.R. and Kareem A.H.A. (2010). Nature of soil-water characteristic curves (SWCC) for soils from Anbar Governorate. Anbar Journal of Engineering Sciences 3(1), 61 - 80

Pham, H.Q. and Fredlund, D.G. (2011). Volume-mass unsaturated soil constitutive for drying-wetting under isotropic loading-unloading conditions. Canadian Geotechnical Journal 48(2), 280-313
Riquelme, J. y Godoy, C. (2016). Determinación experimental de curvas de retención de humedad (SWCC) para ripios y relaves de cobre. IX Congreso Chileno de Ingeniería Geotécnica, Valdivia

Suazo, S., Fourie, A. and Doherty, J. (2016). Experimental study of the evolution of the soil water retention curve for granular material undergoing cement hydration. Journal of Geotechnical and Geoenvironmental Engineering 142(7), 04016022

van Genuchten, M.T. (1980). A closed-form equation for predicting the hydraulic conductivity of unsaturated soils. Soil Science Society of America Journal 44(5), 892 - 898

van Genuchten, M.T., Leij, F.J. and Yates, S.R. (1991). The RETC code for quantifying the hydraulic functions of unsaturated soils. Report by the US Environmental Protection Agency EPA

Vereecken, H., Maes, J., Feyen, J. and Darius, P. (1989). Estimating the soil moisture retention characteristic form texture, bulk density, and carbon content. Soil Science 148(6), 389 - 403 